\title{
Optical properties and optimization of electromagnetically induced transparency in strained InAs/GaAs quantum dot structures
}

Barettin, D.; Houmark-Nielsen, Jakob; Lassen, B.; Willatzen, Morten; Nielsen, Torben Roland; Mørk, Jesper; Jauho, Antti-Pekka

Published in:

Physical Review B Condensed Matter

Link to article, DOI:

10.1103/PhysRevB.80.235304

Publication date:

2009

Document Version

Publisher's PDF, also known as Version of record

Link back to DTU Orbit

Citation (APA):

Barettin, D., Houmark-Nielsen, J., Lassen, B., Willatzen, M., Nielsen, T. R., Mørk, J., \& Jauho, A-P. (2009).

Optical properties and optimization of electromagnetically induced transparency in strained InAs/GaAs quantum dot structures. Physical Review B Condensed Matter, 80(23), 235304.

https://doi.org/10.1103/PhysRevB.80.235304

\section{General rights}

Copyright and moral rights for the publications made accessible in the public portal are retained by the authors and/or other copyright owners and it is a condition of accessing publications that users recognise and abide by the legal requirements associated with these rights.

- Users may download and print one copy of any publication from the public portal for the purpose of private study or research.

- You may not further distribute the material or use it for any profit-making activity or commercial gain

- You may freely distribute the URL identifying the publication in the public portal 


\title{
Optical properties and optimization of electromagnetically induced transparency in strained InAs/GaAs quantum dot structures
}

\author{
D. Barettin* \\ Mads Clausen Institute for Product Innovation, University of Southern Denmark, 6400 Sønderborg, Denmark
}

J. Houmark

DTU Nanotech, Department of Micro- and Nanotechnology, Technical University of Denmark, DK-2800 Kongens Lyngby, Denmark

B. Lassen and M. Willatzen

Mads Clausen Institute for Product Innovation, University of Southern Denmark, 6400 Sønderborg, Denmark

T. R. Nielsen and J. Mørk

DTU Fotonik, Department of Photonics Engineering, Technical University of Denmark, Building 343, DK-2800 Kongens Lyngby, Denmark

A.-P. Jauho

DTU Nanotech, Department of Micro- and Nanotechnology, Technical University of Denmark, DK-2800 Kongens Lyngby, Denmark and Department of Applied Physics, Helsinki University of Technology, P.O. Box 1100, Helsinki FI-02015 HUT, Finland

(Received 21 September 2009; revised manuscript received 3 November 2009; published 2 December 2009)

\begin{abstract}
Using multiband $\vec{k} \cdot \vec{p}$ theory we study the size and geometry dependence on the slow light properties of conical semiconductor quantum dots. We find the $V$-type scheme for electromagnetically induced transparency (EIT) to be most favorable and identify an optimal height and size for efficient EIT operation. In case of the ladder scheme, the existence of additional dipole allowed intraband transitions along with an almost equidistant energy-level spacing adds additional decay pathways, which significantly impairs the EIT effect. We further study the influence of strain and band mixing comparing four different $\vec{k} \cdot \vec{p}$ band-structure models. In addition to the separation of the heavy and light holes due to the biaxial-strain component, we observe a general reduction in the transition strengths due to energy crossings in the valence bands caused by strain and bandmixing effects. We furthermore find a nontrivial quantum dot size dependence of the dipole moments directly related to the biaxial-strain component. Due to the separation of the heavy and light holes the optical transition strengths between the lower conduction and upper most valence-band states computed using one-band model and eight-band model show general qualitative agreement, with exceptions relevant for EIT operation.
\end{abstract}

DOI: 10.1103/PhysRevB.80.235304

PACS number(s): 73.21.La, 78.67.Hc, 42.50.Gy

\section{INTRODUCTION}

InAs/GaAs quantum dot (QD) structures have recently received much attention due to their relevance for optoelectronic devices. ${ }^{1}$ The discrete level nature of quantum dots offer important advantages over bulk and quantum-well material for applications in conventional devices such as lasers and optical amplifiers, as well as devices for all-optical signal processing. However, quantum dots also give the possibility of realizing solid-state implementations of effects and functionalities so far only demonstrated in atomic systems. For instance, the practical exploitation of slow light effects such as electromagnetically induced transparency (EIT) demonstrated in ultracold atoms ${ }^{2}$ is naturally pursued using quantum dots. ${ }^{3}$ Important aspects in this context are to accurately model the optical properties of the quantum dots and to consider the influence of the lattice mismatch induced strain field and its effects on the band structure and eigenstates. Apart from Refs. 4 and 5 that focus on the many-body aspects of EIT operation most theoretical investigations of EIT induced slow light ${ }^{6-9}$ have been based on simple quantum dot models that do not take into account important contributions from, e.g., biaxial strain.
In this paper, we use $\vec{k} \cdot \vec{p}$ theory ${ }^{10,11}$ to determine the band structure of conical quantum dots. A first popular $\vec{k} \cdot \vec{p}$ multiband calculation scheme for bulk materials is due to Luttinger and Kohn ${ }^{12,13}$ which later on was extended to heterostructures by an ad hoc symmetrization procedure. ${ }^{14}$ In order to overcome this ad hoc procedure, Burt formulated the socalled exact envelope-function method ${ }^{15,16}$ and soon after Foreman ${ }^{17}$ used this method to derive a six-band model for the valence bands of zinc-blende heterostuctures. Pokatilov et $a l .{ }^{18}$ have provided an eight-band model for the conduction and the valence bands. They studied quantum dots using a spherical approximation and compared their model, based on exact envelope-function theory, against the usual symmetrized approach. The asymmetry parameter present in the Burt-Foreman formalism but not in the Luttinger-Kohn formalism is shown to lead to changes in approximately $\pm 25 \mathrm{meV}$ in the electronic band structures of InAs/GaAs. ${ }^{18}$

Optoelectronic properties of InGaAs zinc-blende quantum dot with varying shape and size based on $\vec{k} \cdot \vec{p}$ theory have already been studied by Schliwa et al. ${ }^{19}$ and Veprek et al. ${ }^{20}$ However, so far, only a small selection of all possible transitions have been studied. In this work we apply the eightband model based on Burt-Foreman formalism derived in 


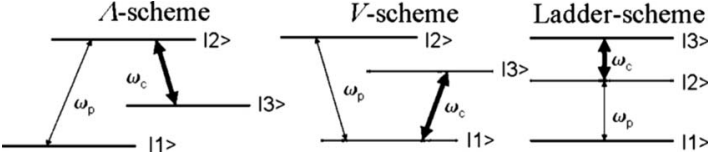

FIG. 1. The three generic EIT schemes. $\omega_{p}$ and $\omega_{c}$ refer to the frequency of the probe and coupling transitions, respectively.

Ref. 18 to zinc-blende InAs/GaAs conical quantum dots. We choose the conical geometry because (i) it introduces important symmetry lowering effects not present in the earlier spherical models and (ii) it mimics closely the structures realized in experimental systems, e.g., in Ref. 21 . We study the size and shape dependence. In particular, we show that some of the, for EIT yet unexplored, interband transitions are highly relevant. Furthermore, we investigate the effect of strain and band mixing between the conduction band and valence bands by comparing four different $\vec{k} \cdot \vec{p}$ models. Although the previous $\vec{k} \cdot \vec{p}$ band-structure studies do include these effects, their impact on optical properties, such as EIT, have not yet been investigated.

The paper is organized as follows. In Sec. II the theory for EIT, band-structure calculations and dipole moments is introduced. In Sec. III we present results for the band-structure and dipole moments calculations, addressing the volume and shape effects while in Sec. IV these results are used to develop level schemes that lead to an efficient EIT operation. The paper is concluded in Sec. V.

\section{THEORY}

\section{A. EIT}

EIT refers to an artificially created spectral region of transparency in the middle of an absorption line due to the destructive quantum interference arising from two transitions in a three-level system. ${ }^{22,23}$ By virtue of the Kramers-Krönig relations a dip in absorption is accompanied by a large positive slope of the refractive index which translates into a reduced group velocity in vicinity of the resonance. Experimental realization of EIT in semiconductor structures has been achieved for the case of quantum wells Refs. 24-27, whereas for QDs, to the best of our knowledge, only a single report exist. ${ }^{28}$

EIT effects in quantum dots and wells have mostly been studied using models that are rooted in atomic physics assuming an archetypical EIT configuration in an ideal medium. ${ }^{3,6-8}$ These models truncate the number of active levels in the system to consider only the three levels being addressed by the laser fields. The generic EIT setup relies on a coupling and a probe laser driving separate transitions (see Fig. 1). In case of the $\Lambda$ scheme, the ground state $|1\rangle$ has the same parity as state $|3\rangle$ and the transition is thereby dipole forbidden. State $|2\rangle$ is of the opposite parity and dipole coupled to both $|1\rangle$ and $|3\rangle$. An intense near resonant continuous-wave electromagnetic field, termed the coupling field, drives the $|2\rangle-|3\rangle$ transition. Another also near resonant, but much weaker probe field, is applied to the $|1\rangle-|2\rangle$ transition.
The archetypical EIT schemes are native to the atomic physics literature and generally one seeks to avoid excitation of carriers. In semiconductors such noncarrier exciting schemes involve an interband probe transition along with an intraband coupling transition. The frequency for the coupling field lies within the deep infrared, a range for which high intensity laser operation is very difficult. Carrier exciting schemes on the other hand involve two interband transitions, where the coupling field is effectively pumping carriers into the conduction band. The carriers interact via the Coulomb force, EIT schemes of this kind have been shown to be favorable when the effect of such many-body interactions are included. ${ }^{4,5,29}$

The study of pulse propagation in a semiconductor slow light medium generally involves solving the coupled Maxwell-Bloch equations (see, e.g., Ref. 30). However, under certain circumstances an analysis of the steady-state properties of the semiconductor Bloch equations (SBEs) alone is adequate. ${ }^{29}$ If this is the case then the linear optical response extracted via the SBE will be directly linked to the propagation characteristics of a wave-packet traveling in an optically thick system.

A relevant figure of merit for slow light operation is the slowdown factor $S$, which is defined as the ratio of the velocity of light in vacuum to the group velocity of a wavepacket traveling through the slow light medium,

$$
S(\omega)=\frac{c_{0}}{v_{g}}=n+\omega \frac{\partial n}{\partial \omega},
$$

where $c_{0}$ is the speed of light in vacuum and $n=\operatorname{Re}\left\{\left[\epsilon_{b}\right.\right.$ $\left.+\chi(\omega)]^{1 / 2}\right\}$ is the refractive index, with $\epsilon_{b}$ being the background permittivity and $\chi(\omega)$ the susceptibility of the active material. The maximum slowdown is found at the frequency for which the slope of the refractive index is largest. Notice that the slowdown factor obtained away from resonance $\left(\frac{\partial n}{\partial \omega} \approx 0\right)$ is given by the background refractive index. The first-order susceptibility, or linear system response, is found from the induced macroscopic polarization $P(\omega)$ as $\chi(\omega)$ $=\frac{P(\omega)}{\epsilon_{0} E_{p}(\omega)}$, where $\epsilon_{0}$ is the vacuum permittivity and $E_{p}(\omega)$ is the amplitude of the probe field. In turn the time-resolved macroscopic polarization component in the direction of the probe field, $P(t)$, is computed from the microscopic polarizations according to semiclassical theory, ${ }^{31}$

$$
P(t)=\frac{1}{w} N_{\mathrm{dot}} \sum_{n, m} \mu_{n m} \rho_{m n}(t),
$$

where $\rho_{n m}$ is the density matrix for localized dot states $(n, m)$. Dipole matrix elements are denoted $\mu_{n m}, N_{\text {dot }}$ is the two-dimensional density of the dots in the growth plane, and $w$ is the thickness of the active region. Within the three-level, (noncarrier exciting) approximation one can derive an analytical expression for the susceptibility $\chi(\omega)$ (see, e.g., Ref. $3)$. When both fields are resonant with their respective transitions $\left(\omega=\omega_{p}\right)$, the slowdown factor $S$ and the absorption $\alpha$ become 


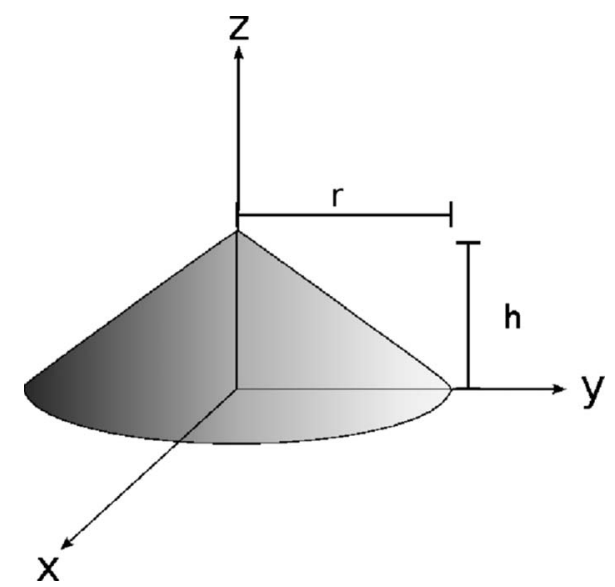

FIG. 2. The shape of the quantum dots under consideration.

$$
\begin{aligned}
S(\omega)= & {\left[\frac{\epsilon_{b}+\sqrt{\epsilon_{b}^{2}+\epsilon_{r e s}^{2}}}{2}\right]^{1 / 2} } \\
& \times\left[1+\frac{\hbar \omega}{2 \sqrt{\epsilon_{b}^{2}+\epsilon_{r e s}^{2}}} \frac{U_{p}\left(\Omega_{c c}^{2}-\gamma_{n r}^{2}\right)}{\hbar^{2}\left(\gamma_{n r} \gamma_{p}+\Omega_{c c}^{2}\right)^{2}}\right], \\
\alpha(\omega)= & \frac{\omega}{c_{0} n_{b}} \operatorname{Im}[\chi(\omega)]=\frac{\omega}{c_{0} n_{b}} \frac{U_{p}}{\hbar} \frac{\gamma_{n r}\left(\Omega_{c c}^{2}+\gamma_{p} \gamma_{n r}\right)}{\left(\Omega_{c c}^{2}+\gamma_{p} \gamma_{n r}\right)^{2}},
\end{aligned}
$$

where $U_{p}=\frac{N_{\mathrm{dot}}}{w}\left|\mu_{p}\right|^{2} / \epsilon_{0}, \quad \Omega_{c c}^{2}=\left|\mu_{c}\right|^{2} I_{c} / 4 \hbar^{2} c \epsilon_{0} \sqrt{\epsilon_{b}}$, and $\epsilon_{\text {res }}$ $=U_{p} / \hbar\left(\gamma_{p}+\Omega_{c c}^{2} / \gamma_{n r}\right) . I_{c}$ is the intensity of the coupling beam, $\mu_{p}$ and $\mu_{c}$ are the dipole moments of the probe and coupling transition, respectively, and $\gamma_{p}$ and $\gamma_{n r}$ are the dephasing rates of the polarization components of the probe and the uncoupled nonradiative transition, respectively. The above expressions display the significance of maximizing the transition strengths of the two light beams. In case of the slowdown factor the dipole moment of the probe transition determines the largest obtainable slowdown while the dipole moment of the pump transition is related to the pump power required to reach this maximum. In an EIT situation $\left(\Omega_{c c}^{2}\right.$ $\left.\gg \gamma_{p} \gamma_{n r}\right)$ the absorption drops as $\omega U_{p} \gamma_{n r} /\left(c_{0} n_{b} \hbar \Omega_{c c}^{2}\right)$, which is proportional to $\left|\mu_{p}\right|^{2} /\left(I_{c}\left|\mu_{c}\right|^{2}\right)$.

\section{B. Band structure}

The dipole moments which govern the EIT characteristics are determined using eight-band $\vec{k} \cdot \vec{p}$ theory including strain effects. We study the conical quantum dots shown in Fig. 2. The dot material is InAs and the barrier material is GaAs. Both InAs and GaAs are zinc-blende materials so in order to reduce the problem to a two-dimensional model we disregard anisotropy effects. This entails that we disregard phenomena such as piezoelectricity and atomistic anisotropic effects. ${ }^{32,33}$ The atomistic anisotropic effects have been investigated by Bester and Zunger ${ }^{34}$ showing that they lead among other things to a splitting of states which in our model are degenerate. This splitting is however less pronounced for cylindrical shaped quantum dots as studied here. Recently it has been shown that second-order piezoelectric terms effectively cancel linear piezoelectric effects for cylindrical shaped quantum dots. ${ }^{19,35,36}$ We have checked the isotropic assumption and found a maximum error for the strain fields of $8 \%$ along the $z$ axis, going rapidly to zero at the edges of the dot.

We investigate both the effect of strain and band mixing. The effect of band mixing is determined by comparing the eight-band results with one-band model results for the conduction band and the heavy holes. Due to the lattice mismatch present in the systems under consideration in this paper (InAs/GaAs quantum dots) the materials will be strained. In the eight-band model the wave function $\psi_{n}$ of the localized dot state $n$ is given by a linear combination of the eight Bloch states weighted by an envelope function,

$$
\psi_{n}=\sum_{i=1}^{8} \phi_{i}^{(n)} u_{i},
$$

where $\phi_{i}^{(n)}$ are the envelope function and $u_{i}$ are the Bloch states. We use the eight-band Hamiltonian described in Ref. 18 while for the strain-dependent part we follow Ref. 37.

In the following we explicitly present the one-band models as due to their simplicity these are most open to interpretation. The one-band eigenvalue equation for the envelope functions reads $H_{\alpha} \phi_{\alpha}=E_{\alpha} \phi_{\alpha}$, where $H_{\alpha}, \phi_{\alpha}$, and $E_{\alpha}$ are the Hamiltonian, the envelope wave function, and the energy, respectively. $\alpha$ denotes either the conduction band or the heavy holes, i.e., $\alpha=\{e, h h\}$. The Hamiltonian is

$$
H_{\alpha}=H_{\alpha}^{k}\left(\overrightarrow{r_{\alpha}}\right)+H_{\alpha}^{b}\left(\overrightarrow{r_{\alpha}}\right)+H_{\alpha}^{\epsilon}\left(\overrightarrow{r_{\alpha}}\right)
$$

where $H_{\alpha}^{k}\left(\vec{r}_{\alpha}\right)$ is the kinetic part, $H_{\alpha}^{b}\left(\vec{r}_{\alpha}\right)$ is the energy of the unstrained band edge, and $H_{\alpha}^{\epsilon}\left(\vec{r}_{\alpha}\right)$ is the strain-dependent part. The solutions are spin degenerate in the one-band model, i.e., $\psi_{e}^{\uparrow}=\phi_{e}|S \uparrow\rangle$ and $\psi_{e}^{\downarrow}=\phi_{e}|S \downarrow\rangle$, and $\psi_{h h}^{\uparrow}=\phi_{h h}|h h \uparrow\rangle$ and $\psi_{h h}^{\downarrow}=\phi_{h h}|h h \downarrow\rangle$.

In the one-band model the strain Hamiltonian for a zincblende crystal structure is given by

$$
\begin{gathered}
H_{e}^{\epsilon}\left(\overrightarrow{r_{e}}\right)=a_{c}(\vec{r}) \varepsilon_{H}(\vec{r}), \\
H_{h h}^{\epsilon}\left(\overrightarrow{r_{h h}}\right)=-a_{v}(\vec{r}) \varepsilon_{H}(\vec{r})+\frac{b}{2} \varepsilon_{B}(\vec{r}),
\end{gathered}
$$

where $a_{c}\left(a_{v}\right)$ is the conduction (valence)-band hydrostatic deformation potential and $b$ is the shear deformation potential $^{38}$ while the hydrostatic and biaxial-strain components read

$$
\begin{aligned}
& \varepsilon_{H}(\vec{r})=\varepsilon_{x x}(\vec{r})+\varepsilon_{y y}(\vec{r})+\varepsilon_{z z}(\vec{r}), \\
& \varepsilon_{B}(\vec{r})=\varepsilon_{x x}(\vec{r})+\varepsilon_{y y}(\vec{r})-2 \varepsilon_{z z}(\vec{r}),
\end{aligned}
$$

where $\varepsilon_{i k}$ is the strain tensor. The strain fields are found by minimizing the elastic strain energy. ${ }^{32}$

\section{Dipole moments}

The momentum matrix element $\vec{p}_{n m}$ is given by 

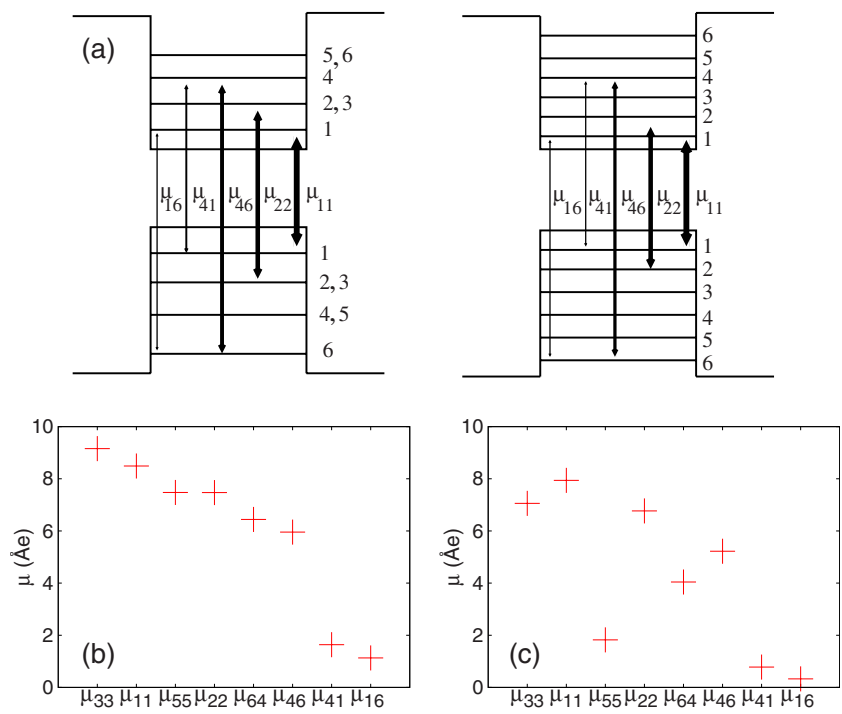

FIG. 3. (Color online) Energy levels and the most relevant dipole moments for EIT (top) and values of the eight strongest interband dipole moments (bottom) for one-band (left) and eight-band (right) model without strain for a dot with $h=7.5 \mathrm{~nm}$.

$$
\begin{aligned}
\vec{p}_{n m} & \equiv\left\langle\psi_{n}|\vec{p}| \psi_{m}\right\rangle, \\
& =\sum_{i, j=1}^{N}\left(\left\langle\phi_{i}^{(n)}|\vec{p}| \phi_{j}^{(m)}\right\rangle \delta_{i j}+\left\langle\phi_{i}^{(n)} \mid \phi_{j}^{(m)}\right\rangle\left\langle u_{i}|\vec{p}| u_{j}\right\rangle\right), \\
& \equiv \vec{p}_{n m}^{(\phi)}+\vec{p}_{n m}^{(u)},
\end{aligned}
$$

where $N=1$ or $8 . \vec{p}^{(\phi)}$ and $\vec{p}^{(u)}$ are the envelope and the Bloch parts of the momentum matrix element, respectively. It is usual to consider only the Bloch part $\vec{p}^{(u)}$ since the envelope part $\vec{p}^{(\phi)}$ is usually an order of magnitude smaller. ${ }^{39}$

As shown in Ref. 40, the evaluation of the momentum matrix element $\vec{p}_{n m}$ is meaningful while dipole-matrix $\vec{\mu}_{n m}$ elements are ill defined in crystals involving extended Bloch states. Thus, we first calculate $\vec{p}_{n m}$ and then use the relation between the momentum and the electric dipole-matrix element given by Ref. 41

$$
\vec{p}_{n m}=\frac{i m_{0}}{e} \omega_{n m} \vec{\mu}_{n m},
$$

where $\hbar \omega_{n m}=E_{n}-E_{m}, m_{0}$ is the free-electron mass and $e$ is the electronic charge. For material parameters used in calculations we refer to Ref. 42.

\section{RESULTS: BAND STRUCTURE AND DIPOLE MOMENTS}

The first results we present are related to a set of conical quantum dots where the aspect ratio between the radius $r$ and the height $h$ of the dot has been fixed so that $r=2 h$. We focused on the first 12 bound states for both bands. Since all the states are at least doubly degenerate (spin degeneracy), we consider six energy levels (labeled from 1 to 6) for both bands. In the one-band model, due to the conical quantum dot symmetry and isotropy assumption (giving an inversion symmetric model), level 2 and level 3 are degenerate for
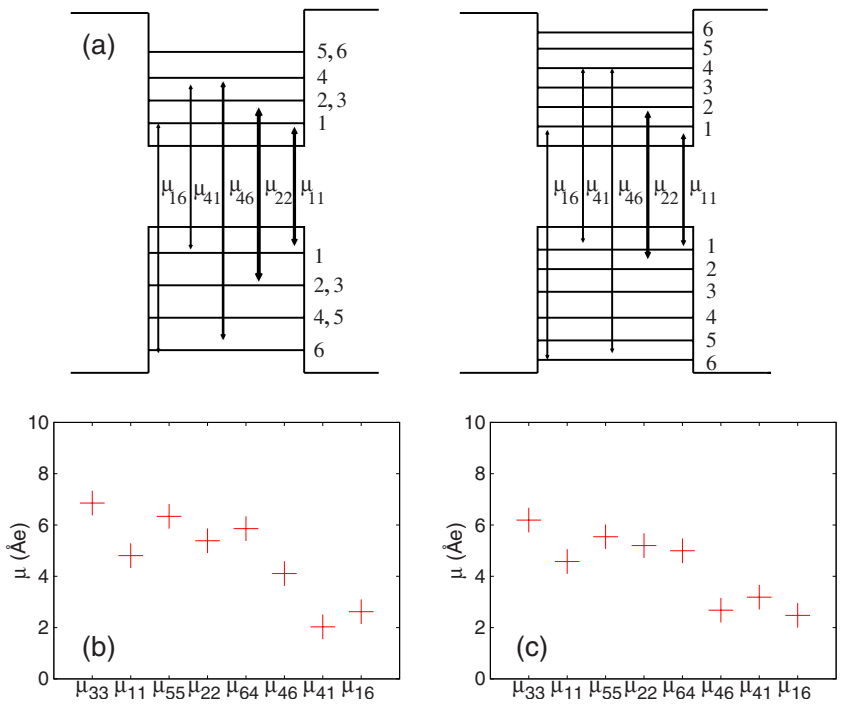

FIG. 4. (Color online) Energy levels and the most relevant dipole moments for EIT (top) and values of the eight strongest interband dipole moments (bottom) for one-band (left) and eight-band (right) model with strain for a dot with $h=7.5 \mathrm{~nm}$.

both the conduction and the valence band, level 4 and level 5 are degenerate for the valence band, and level 5 and level 6 are degenerate for the conduction band. In the upper part of Figs. 3 and 4 we show the energy levels and the most relevant interband dipole moments for EIT ( $\mu_{22}$ is also included due to its relevance for other applications) corresponding to a dot with $h=7.5 \mathrm{~nm}$ for the four different models: one-band model without strain, one-band model with strain, eight-band model without strain, and eight-band model with strain. Throughout this paper we consider only right-handed circularly polarized light.

In Figs. 3 and 4 the thicknesses of the lines indicating the transitions are proportional to the corresponding dipole moments. Obviously, an eight-band model calculation leads to a higher valence-band density of states as compared to a oneband calculation. Hence, also more interband transitions result, in a given energy range, when using an eight-band model. We have chosen to show in the bottom part of Figs. 3 and 4 only the strongest eight dipole-matrix elements for both one-band and eight-band models. First, we observe that there is a qualitative agreement between dipole-moment results for the one-band and eight-band models with strain (Fig. 4). This is due to the fact that in the eight-band model the biaxial-strain component of Eq. (10) shifts heavy holes (light holes) to higher (lower) energies. As a consequence the valence-band ground state and the first excited states are predominantly heavy holes like giving rise to a general better agreement between the one-band model and the eight-band model. The only notable discrepancies are observed for the EIT relevant transitions $\mu_{46}$ and $\mu_{41}$. Second, the inclusion of strain reduces the strength of the dipole moments significantly. This is because there is a nontrivial influence of strain on dipole moments. The conduction-band states are only affected by the hydrostatic strain component of Eq. (9) giving rise more or less to a constant shift in the effective potential inside the dot while the valence-band states, in addition to 


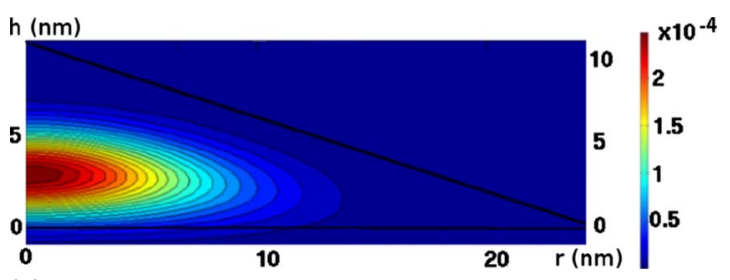

(a)

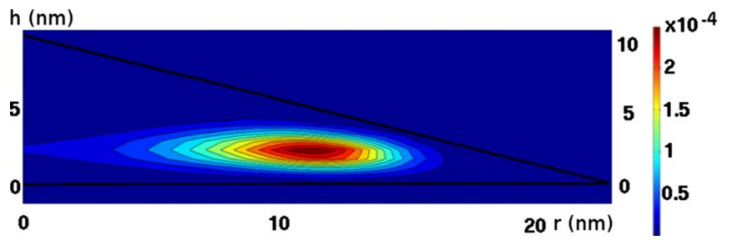

(b)

FIG. 5. (Color online) Probability density $|\psi|^{2}$ of the valenceband ground state for the eight-band model without (up) and with (down) strain. The dimensions of the quantum dot are $h=11.5 \mathrm{~nm}$ and $r=23 \mathrm{~nm}$.

the hydrostatic strain, are also affected by the biaxial-strain component [see Eq. (10)]. The latter component is highly inhomogeneous inside the dot. In the eight-band model there is a third contribution from $\epsilon_{x z}$ and $\epsilon_{y z}$ strain components ${ }^{37}$ but this term is not as significant as the biaxial of Eq. (10).

In order to understand the influence of strain on the dipole moment we compare in Fig. 5 the valence-band ground-state probability density $|\psi|^{2}$ (eight-band model) with and without the influence of the strain field for a quantum dot with height $h=11.5 \mathrm{~nm}$ and radius $r=23 \mathrm{~nm}$. While in the case without strain the ground state shows a $s$-like shape, the biaxial-strain component modifies the hole wave function into a toroidal shape moving it away from the center of the dot where the potential is stronger. This drastically reduces the overlap between the envelope functions $\phi_{i}$ of the conduction and valence band and consequently the corresponding dipole moments.

The reduction in the dipole moment due to strain is evident in Fig. 6 where we plot the interband dipole moment $\mu_{11}$ between the conduction-band and the valence-band

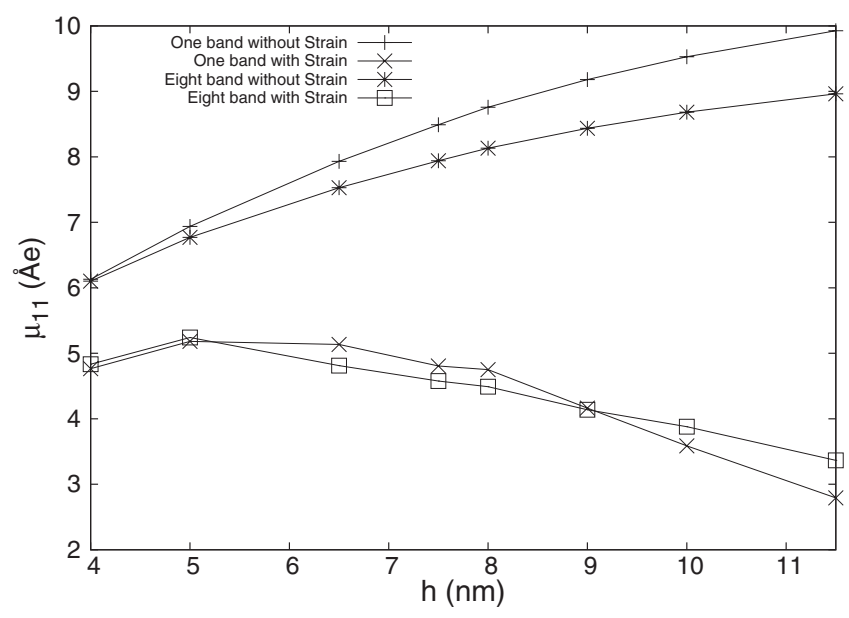

FIG. 6. Interband $\mu_{11}$ dipole moments as a function of $h$ for the four different models.

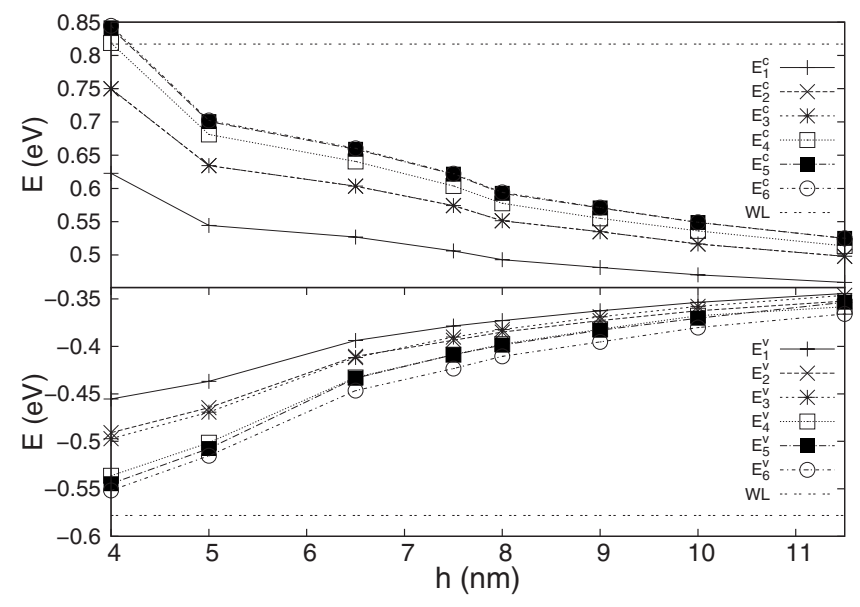

FIG. 7. Energies of the six considered levels in conduction (top) and valence (bottom) band as a function of $h$ for eight-band model with strain. The onset of continuous WL states is also indicated (dashed-horizontal line).

ground states for the four different models as a function of $h$. In the models with strain we have a maximum around $h$ $=5 \mathrm{~nm}$ while the dipole moments for the two models without strain grow monotonically with increasing height and eventually reach a plateau value corresponding to the bulk value of $16.8 \AA$ Ae. The monotonic increase in the dipole moments can be understood based on Eq. (12). Without strain the momentum matrix elements $\vec{p}$ (mainly determined by $\vec{p}^{(u)}$ ) remains constant with increasing height whereas the energy difference $\omega_{n m}$ decreases. The presence of strain reduces the overlap of electron and hole distributions as a result of the increased displacement of the hole wave functions away from the center leading to the observed decrease in the dipole moments.

In Fig. 7 we plot the energies of the first six levels in the conduction (top) and valence band (bottom) as a function of the height $h$. The wave functions of the confined states are characterized (in the eight-band model) by eight envelopes weighted differently depending on the state. As mentioned above, the biaxial strain is inhomogeneous and this, combined with the differently spatially distributed envelope functions, leads to a higher sensitivity against strain as compared to a one-band model. Further, the inhomogeneity of the strain field grows with volume, especially for the biaxial-strain component of Eq. (10). These coupled strain-band-mixing effects lead to energy crossings in the valence band. We have also indicated where the wetting-layer (WL) continuum starts. The WL dashed lines in Fig. 7 are computed using a Ben-Daniel Duke approach for a $0.5 \mathrm{~nm}$ InAs quantum well embedded in GaAs. ${ }^{14}$ We observe that only the last three considered conduction-band levels for the smallest quantum dot lay above the lower bound of the wetting-layer continuum states.

The most relevant dipole moments for EIT for the eightband model are shown in the upper part of Fig. 8 as a function of $h$. The bottom part gives the related energy differences $\Delta E$. The strain reduces the dipole-moment strengths with increasing volume because of the decreasing wave function overlap (similar to what was found for $\mu_{11}$ ). 


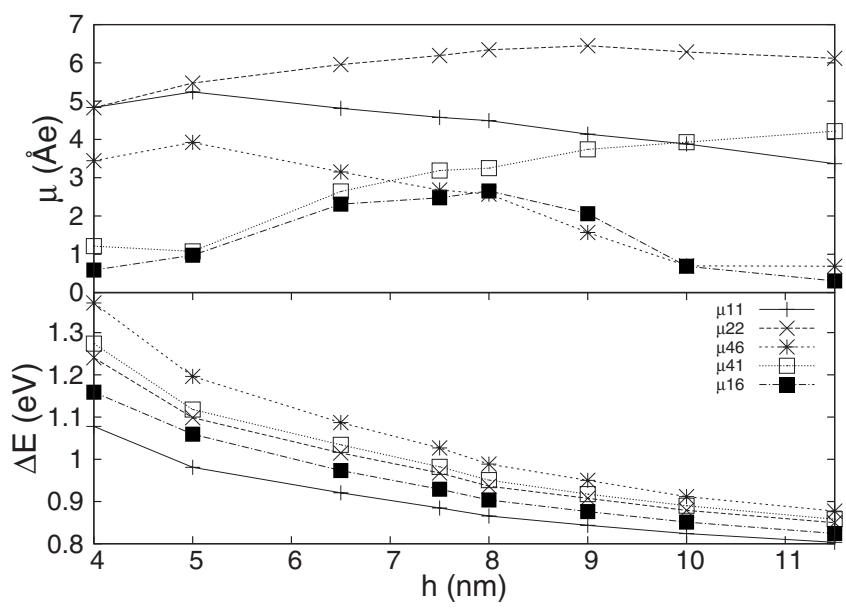

FIG. 8. Interband dipole moments $\mu$ (top) and relative band energy difference $\Delta E$ (bottom) as a function of the height $h$ with fixed aspect ratio $r=2 h$.

This geometry effect is indeed mainly a function of the dot volume as Fig. 9 shows. Here, we refer to a second set of quantum dots with different aspect ratios $\left(A_{s p}=r / h\right)$ having the same volume $\left[V=226.19(\mathrm{~nm})^{3}\right]$. The interband dipole moments (top) and relative energy differences (bottom) are depicted as a function of $A_{s p}$ and evidently results are rather insensitive to the aspect ratio.

\section{RESULTS: EIT}

\section{A. Three levels vs many levels}

In this section we consider right-handed circularly polarized light for both the coupling and probe fields and investigate the slow light characteristics upon propagation through several stacked layers of QD material. We assume that one can disregard propagation effects in the coupling field. In case of noncarrier exciting schemes, the coupling field is effectively connecting two empty states, thus rendering the transition transparent. In the case where the coupling beam is exciting carriers we consider a propagation through sufficiently thin device so that the coupling intensity remains constant. We use a lattice temperature of $200 \mathrm{~K}$, for which the literature ${ }^{43,44}$ gives dephasing rates around 1.5 $\times 10^{12} \mathrm{~s}^{-1}$. The system response is calculated using a procedure similar to Ref. 29. First we investigate a ladder-type scheme. The ladder scheme is generally noncarrier exciting. We consider a situation where the probe pulse connects the conduction-band/valence-band ground state $\left(\mu_{11}\right)$ and the coupling beam is tuned to the intraband transition in the conduction band between the ground state and first excited level. This particular setup has been the prime candidate for theoretical scrutiny ${ }^{3,8}$ employing a three-level approach along with a single-band model including only hydrostatic strain. To illustrate the importance of including all energy levels and transitions along with a more detailed bandstructure calculation we show in Fig. 10 the imaginary part of the susceptibility calculated using a coupling intensity of $1 \mathrm{MW} / \mathrm{cm}^{2}$ for two dot sizes (height 7.5 and $9 \mathrm{~nm}$, both $A_{s p}=2$ ) using either the most simple model (one-band un-

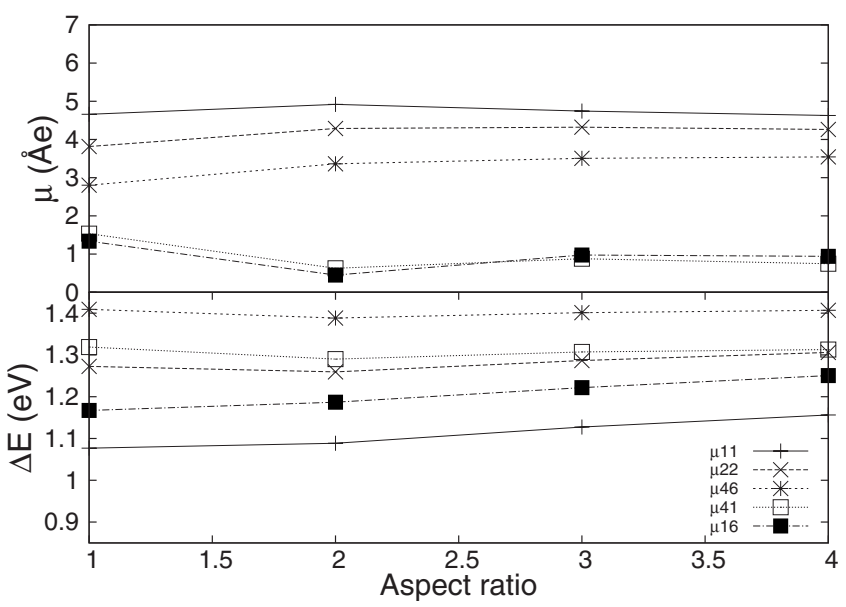

FIG. 9. Interband dipole moments $\mu$ (top) and relative band energy difference $\Delta E$ (bottom) as a function of the aspect ratio at constant volume.

strained) or the most complex (eight-band strained).

Most notably the EIT effect, which is recognized as a dip in the absorption spectrum symmetric around the probe frequency and is present in the simple three-level models, is absent from three out of four multilevel calculations. The reason for this is that the additional level structure is dipole coupled to the three-level EIT system. In the particular ladder configuration considered here, due to the relatively close spacing between the energy levels, higher lying states are also being addressed by the coupling field, thus introducing additional transitions and thereby adding alternative decay pathways that effectively remove the destructive interference between the available paths, which is at the origin of EIT. In the one band unstrained calculation the energy levels are almost equidistantly spaced resulting in a particularly pathologic case, namely, a peak instead of a dip in the spectrum. However, by detuning the coupling frequency one can filter out the contributions from higher lying states and thus restore a resemblance to the three-level symmetric case. This is indirectly evidenced in the multiband calculations including strain. Here the energy-level structure is modified, the level structure is no longer equidistantly spaced, and as a consequence the additional transitions are now detuned with respect to the coupling field. In fact the multilevel model for the dot of $7.5 \mathrm{~nm}$ height indeed displays the EIT effect and produces a spectrum similar to the simple three-level treatment. The eight-band multilevel calculation for the larger dot $(h=9 \mathrm{~nm})$ has reminiscence of the EIT effect but the spectrum is quite far from the "ideal" spectrum predicted by the simple model. Again, this can be remedied by detuning the coupling field. In general, ladder schemes in rotationally symmetric dots are, due to the near equidistantly spaced energy levels, likely subjected to the issue of the coupling beam being in resonance with other transitions. Carrier exciting $\Lambda$ or $V$-schemes connecting only interband transitions are due to their larger transition energies much less likely to encounter this problem. Furthermore, the frequency range of interband transitions is much easier accessible to industry lasers than the near infrared required by ladder-type schemes. 

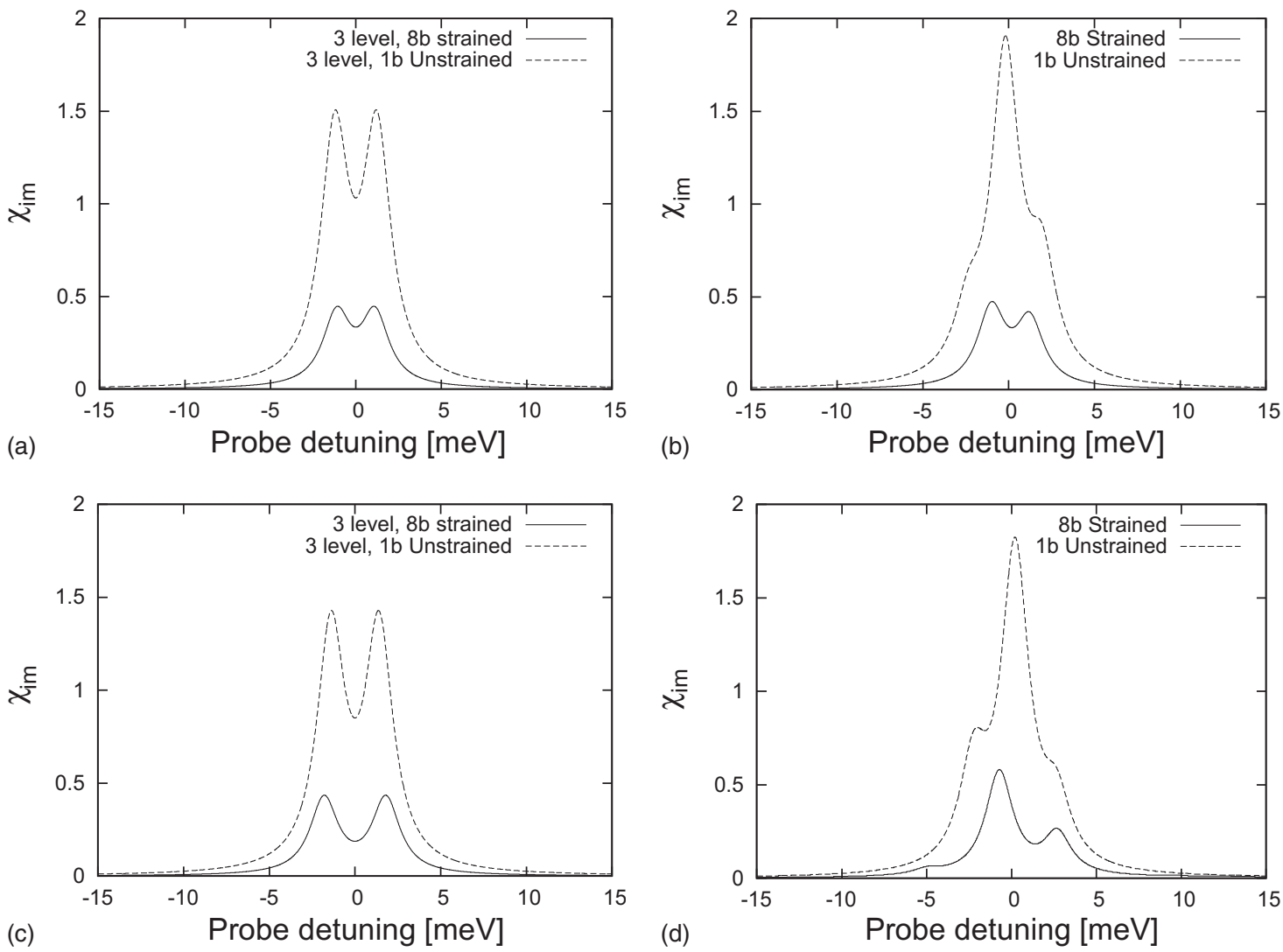

FIG. 10. Example of susceptibility calculated for a ladder scheme using the three levels or a full multilevel approach based on either the eight-band strained or one-band unstrained model. The results are presented for dots of height $7.5 \mathrm{~nm}$ (top panel) and $9 \mathrm{~nm}$ (bottom panel), both $A_{s p}=2$. The reason for the larger features in the one-band calculations is that the strength of the probe transition is larger within this model (see Fig. 6).

\section{B. Size/geometry dependence}

The possible interband EIT schemes are illustrated in Fig. 4 (top). By combining the shown EIT relevant transitions a variety of different $V$ as well as $\Lambda$ schemes can be accessed. As noted in a previous section there are very few differences between dipole moments predicted by the one-band strained model and the eight-band strained model, however the deviations that do exist, appears for transitions that could be used in connection with EIT. The one-band strained model significantly overestimates $\mu_{46}$, a transition that would otherwise seem attractive to use for EIT. Furthermore $\mu_{41}$ is very small in all but the eight-band strained model. To illustrate the impact of these differences we have in Fig. 11 shown absorption spectra for an EIT scheme constructed using $\mu_{41}$ as probe and $\mu_{46}$ as coupling transition. It is seen that using eight-band strained band-structure results in an altered EIT behavior. The signature feature, the split peaks, is higher and closer together, a direct consequence of the magnitudes of the involved dipole moments. These significant differences between the spectra justifies focusing on the results of the more exact eight-band strained band-structure model. $V$ and $\Lambda$ schemes can be realized using $\mu_{41}$ as coupling transition and either of the two transitions $\mu_{11}$ or $\mu_{46}$ for the probe field. Two similar schemes can be accessed using the same probe transitions and $\mu_{16}$ for the coupling field. As is evident from Fig. 9 the dipole moments are relatively unchanged by varying shape (aspect ratio), we choose to focus on $A_{s p}=2$ since the common probe transitions $\left(\mu_{11}\right.$ and $\left.\mu_{46}\right)$ are maximized for this geometry and consider volume dependencies.

We see that for small dots $(h<5 \mathrm{~nm})$ the dipole moments addressed by the coupling beam are relatively small (on the order of $1 \mathrm{~nm}$ ) and therefore dots of this size are not suitable for EIT operation. As the dot increases in size both coupling

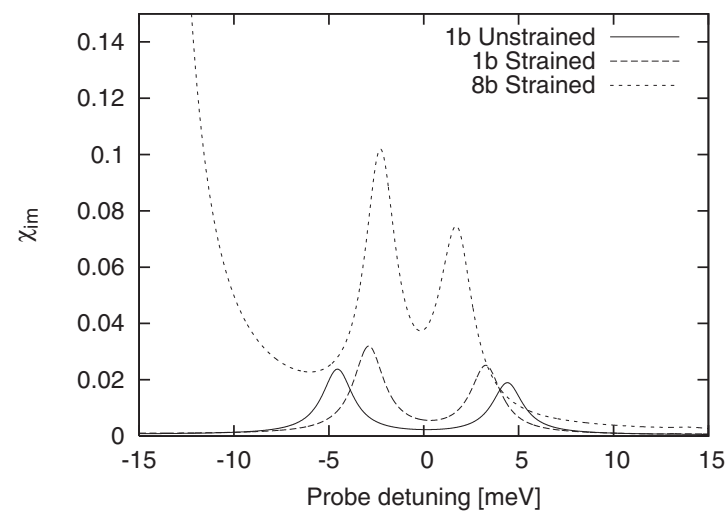

FIG. 11. EIT absorption spectrum calculated using $\mu_{41}$ as probe and $\mu_{46}$ as coupling transition for different models: one-band unstrained (full line), one-band strained (dashed line), and eight-band strained (dotted line). The additional feature in the eight-band strained calculation is due to the nearby lying $\mu_{55}$ resonance. 


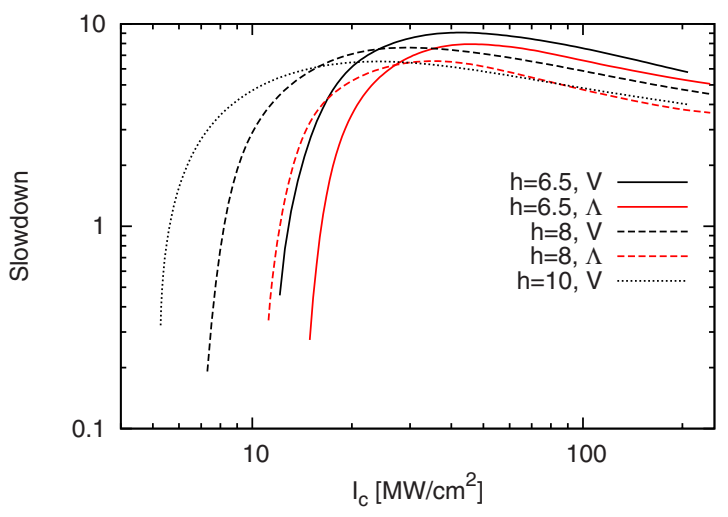

FIG. 12. (Color online) Slowdown versus coupling beam intensity calculated for three different dot sizes. The black curve refers to the $V$ scheme whereas the red curve is for $\Lambda$ configuration.

transitions become stronger, the two probe transitions, however begins to decrease. The dipole $\mu_{46}$ is always the smaller of the two probe transitions and the fact that it drops off faster suggests that one should aim at using $\mu_{11}$, ground state to ground state, as probe transition.

To quantify the above discussion we show in Fig. 12 the slowdown factor as a function of coupling intensity calculated for three different dot volumes $(h=6.5,8$, and $10 \mathrm{~nm})$ for each of the two remaining EIT schemes (for the $h$ $=10 \mathrm{~nm}$ dot, in the case of the $\Lambda$ scheme no EIT behavior is found due to its weak-coupling transition. Slowdown results for this dot/EIT setup has therefore been omitted in Fig. 12). For every dot type we see that the $V$ scheme prevails, it has the lowest coupling power requirements and the largest obtainable slowdown value. The first observation can be understood from Fig. 8 where the $V$-scheme coupling transition $\mu_{41}$ is always the larger. Since both schemes utilize the same probe transition one could be inclined to think that they should display the same maximum slowdown value. The reason they do not is that the coupling beam, although detuned, is exciting carriers into the levels connected by the probe beam. The presence of the carriers serves to block the probe transition. As indicated in the lower part of Fig. 8, the cou- pling beam in the $\Lambda$ case is less detuned from the probe transition than in the $V$ case, and thus more carriers are excited into the electron and hole ground states, resulting in a smaller effective probe transition strength. It is apparent that the best tradeoff between maximum slowdown and coupling intensity is found using the $V$ scheme for dots having a relatively large height, in the region around $8 \mathrm{~nm}$. In the literature the $V$ scheme has also been deemed preferable due to its ability to overcome inhomogeneous broadening ${ }^{9}$ and a favorable carrier redistribution mechanism. ${ }^{29}$

\section{CONCLUSION}

For the investigated system we have shown that the strain field generally reduces optical transition strengths as a function of the volume of the quantum dot for a fixed aspect ratio. This is due to a decreasing overlap of the involved wave functions. This geometry effect has been shown to be a volume effect rather than a shape effect. Moreover, the combined influence of band mixing and strain entails state crossings in the valence band, and a separation of the heavy and light holes. The latter leads to a qualitative agreement between the lower conduction and upper most valence-band states computed using the one-band model and the eightband model. The observed discrepancies for $\mu_{46}$ and $\mu_{41}$ have an impact on the choice of EIT scheme. We have investigated the importance of including the full energy-level structure and optical transitions in an EIT simulation. In particular, we find for a ladder-type scheme that the additional transitions along with an almost equidistant energy-level spacing can severely impair the EIT effect. We have studied the effect of varying dot size and geometry on EIT and identified a $V$-type configuration in a dot of aspect ratio $r=2 h$ with height $h \approx 8 \mathrm{~nm}$ to have the best possibilities for efficient EIT operation.

\section{ACKNOWLEDGMENTS}

This work is supported by the Danish Research Council for Technology and Production (QUEST). A.P.J. is grateful to the FiDiPro program of the Finnish Academy.

\footnotetext{
*Corresponding author; daniele@mci.sdu.dk

${ }^{1}$ J. Piprek, Nitride Semiconductor Devices-Principles and Simulation (Wiley VHC, New York, 2007).

${ }^{2}$ L. V. Hau, S. E. Harris, Z. Dutton, and C. H. Behroozi, Nature (London) 397, 594 (1999).

${ }^{3}$ C. J. Chang-Hasnain, P. C. Ku, J. Kim, and S. L. Chuang, Proc. IEEE 91, 1884 (2003).

${ }^{4}$ S. Michael, W. W. Chow, and H. C. Schneider, Appl. Phys. Lett. 89, 181114 (2006).

${ }^{5}$ W. W. Chow, S. Michael, and H. C. Schneider, J. Mod. Opt. 54, 2413 (2007).

${ }^{6}$ J. Kim, S. L. Chuang, P. C. Ku, and C. J. Chang-Hasnain, J. Phys.: Condens. Matter 16, S3727 (2004).

${ }^{7}$ P. Jänes, J. Tidström, and L. Thylén, J. Lightwave Technol. 23,
}

3893 (2005).

${ }^{8}$ P. K. Nielsen, H. Thyrrestrup, J. Mørk, and B. Tromborg, Opt. Express 15, 6396 (2007)

${ }^{9}$ P. Lunnemann and J. Mørk, Appl. Phys. Lett. 94, 071108 (2009).

${ }^{10}$ E. Kane, Physics of III-V Compounds (Academic, New York, 1966), Vol. 1, Chap. 3.

${ }^{11}$ G. Dresselhaus, A. F. Kip, and C. Kittel, Phys. Rev. 98, 368 (1955).

${ }^{12}$ J. M. Luttinger, Phys. Rev. 102, 1030 (1956).

${ }^{13}$ J. M. Luttinger and W. Kohn, Phys. Rev. 97, 869 (1955).

${ }^{14}$ G. Bastard, Wave Mechanics Applied to Semiconductor Heterostructures (les Editions de Physique, Les Ulis Cedex, France, 1988).

${ }^{15}$ M. G. Burt, J. Phys.: Condens. Matter 4, 6651 (1992). 
${ }^{16}$ M. G. Burt, Phys. Rev. B 50, 7518 (1994).

${ }^{17}$ B. A. Foreman, Phys. Rev. B 48, 4964 (1993).

${ }^{18}$ E. P. Pokatilov, V. A. Fonoberov, V. M. Fomin, and J. T. Devreese, Phys. Rev. B 64, 245328 (2001).

${ }^{19}$ A. Schliwa, M. Winkelnkemper, and D. Bimberg, Phys. Rev. B 76, 205324 (2007).

${ }^{20}$ R. G. Veprek, S. Steiger, and B. Witzigmann, J. Comput. Electron. 7, 521 (2008).

${ }^{21}$ T. Inoue, T. Kita, O. Wada, M. Konno, T. Yaguchi, and T. Kamino, Appl. Phys. Lett. 92, 031902 (2008).

${ }^{22}$ S. E. Harris, J. E. Field, and A. Imamoglu, Phys. Rev. Lett. 64, 1107 (1990).

${ }^{23}$ S. E. Harris, Phys. Today 50(7), 36 (1997).

${ }^{24}$ G. B. Serapiglia, E. Paspalakis, C. Sirtori, K. L. Vodopyanov, and C. C. Phillips, Phys. Rev. Lett. 84, 1019 (2000).

${ }^{25}$ M. C. Phillips and H. Wang, Phys. Rev. B 69, 115337 (2004).

${ }^{26}$ H. Kang, J. S. Kim, S. I. Hwang, Y. H. Park, D. kyeong Ko, and J. Lee, Opt. Express 16, 15728 (2008).

${ }^{27}$ S.-M. Ma, H. Xu, and B. S. Ham, Opt. Express 17, 14902 (2009).

${ }^{28}$ S. Marcinkevičius, A. Gushterov, and J. P. Reithmaier, Appl. Phys. Lett. 92, 041113 (2008).

${ }^{29}$ J. Houmark, T. R. Nielsen, J. Mørk, and A.-P. Jauho, Phys. Rev. B 79, 115420 (2009).

${ }^{30}$ T. R. Nielsen, A. Lavrinenko, and J. Mørk, Appl. Phys. Lett. 94, 113111 (2009).
${ }^{31} \mathrm{H}$. Haug and S. W. Koch, Quantum Theory of the Optical and Electronic Properties of Semiconductors, 3rd ed. (World Scientific, Singapore, 1990).

${ }^{32}$ V. A. Fonoberov and A. A. Balandin, J. Appl. Phys. 94, 7178 (2003).

${ }^{33}$ D. Barettin, B. Lassen, and M. Willatzen, J. Phys.: Conf. Ser. 107, 012001 (2008).

${ }^{34}$ G. Bester and A. Zunger, Phys. Rev. B 71, 045318 (2005).

${ }^{35} \mathrm{G}$. Bester, A. Zunger, X. Wu, and D. Vanderbilt, Phys. Rev. B 74, 081305(R) (2006).

${ }^{36} \mathrm{G}$. Bester, X. Wu, D. Vanderbilt, and A. Zunger, Phys. Rev. Lett. 96, 187602 (2006).

${ }^{37}$ Y. Zhang, Phys. Rev. B 49, 14352 (1994).

${ }^{38}$ G. L. Bir and G. E. Pikus, Symmetry and Strain-Induced Effects in Semiconductors (Wiley, New York, 1974).

${ }^{39}$ O. Stier, M. Grundmann, and D. Bimberg, Phys. Rev. B 59, 5688 (1999).

${ }^{40}$ M. G. Burt, J. Phys.: Condens. Matter 5, 4091 (1993).

${ }^{41}$ E. Merzbacher, Quantum Mechanics (Wiley, New York, 1961).

${ }^{42}$ I. Vurgaftman, J. R. Meyer and L. R. Ram-Mohan, J. Appl. Phys. 89, 5815 (2001).

${ }^{43}$ P. Borri, W. Langbein, J. Mørk, J. M. Hvam, F. Heinrichsdorff, M. H. Mao, and D. Bimberg, Phys. Rev. B 60, 7784 (1999).

${ }^{44}$ P. Borri, W. Langbein, S. Schneider, U. Woggon, R. L. Sellin, D. Ouyang, and D. Bimberg, Phys. Rev. Lett. 87, 157401 (2001). 